\title{
Introducing Patient and Dentist Profiling and Crowdsourcing to Improve Trust in Dental Care Recommendation Systems
}

\author{
Sojen Pradhan and Valerie Gay \\ University of Technology Sydney (UTS) \\ Sydney, Australia \\ \{Sojendra.Pradhan, Valerie.Gay\}@uts.edu.au
}

\begin{abstract}
Healthcare blogs, podcasts, search engines and health social networks are now widely used, and referred as crowdsources, to share information such as opinions, side effects, medication and types of therapies. Although attitudes and perceptions of the users play a vital role on how they create, share, retrieve and utilise the information for their own or recommend to others, recommendation systems have not taken the attitudes and perceptions into considerations for matching. Our research aims at defining a trust dependent framework to design recommendation system that uses profiling and social networks in dental care. This paper focuses on trust derived in direct interaction between a patient and a dentist from subjective characteristics' point of view. It highlights that attitudes, behaviours and perception of both patients and dentists are important social elements, which enhance trust and improve the matching process between them. This study forms a basis for our profile-based framework for dynamic dental care recommendation systems.
\end{abstract}

Keywords: Health social networks, Social communications, Crowdsourcing, Recommendation systems, Trust, Reviews and ratings.

\section{Introduction}

It is part of the human nature to share experiences, and to turn to our peers to get support, recommendations and answers on all aspects of our life. This feature has been adopted online, and it is done through various channels such as blogs, micro blogs, wikis, forums, social networking sites (SNSs). The source of information is gradually moving from friends, families, radio and television to the Internet. Web users check specific websites before making decisions about their everyday activities (what to buy, where to eat, which movie to watch, which professionals to choose). Social networks such as Amazon, eBay, IMDb, Eatability, TripAdvisor, Epinion, Elance, and also Health social networks (HSNs) such as PatientsLikeMe, CureTogether, HealthLine, DailyStrength, WebMD are providing recommendations to the users.

One of the evolving features of the online world is 'reviews' and 'ratings' on any product or service. Crowdsources have been influencing the users in making daily 
decisions to choose a particular product or service, beyond the traditional form of instructions from experts or local peers or health professionals [1,2]. These reviews and ratings are shaping and influencing public views on health issues such as vaccines, breast implants and dental implants. Thus, sources of health information is shifting online. Moreover, HSNs are promoting a sense of community and providing variety of services such as emotional support, self-tracking, Physician Q\&A and clinical trial access [3], to win trust from the web users.

Trust is a complex phenomenon that is used in many aspects of our daily life. Whenever a truster interacts with a trustee, a level of trust is inherently present regardless of simple or complex types of interactions [4]. The level of trust means the level of confidence of a truster in the trustee to act or carry on the action in a given situation. In this paper, we are focusing on how a level of trust changes with different types of people with their personality, attitude, taste and perception [5] and how it can be captured within a HSN environment. Our research aims at designing a framework for dynamic recommendation systems in dental care by including social elements of trust from patients' and dentists' profiles. Trust on the recommendation system as an information provider for patients, would be increased.

This paper is organised as follows; Section 2 discusses a shift of social communication and rise of recommendation systems. It is followed by a study of the complex nature of trust in Section 3. Section 4 proposes a framework for dental care recommendations systems based on trust enhanced by profiling both patients and dentists. Trust measurement through patients and dentists profiles are further elaborated in section 5 . We conclude this paper outlining future works to validate the framework.

\section{Social Communications and Recommendation Systems}

Some studies [6,7] highlighted the fact that we live in a 'small world', and demonstrated that two people in the US are connected by less than 'six degrees of separation'. With the rise of social media, a study claims that the degrees of separation have reduced to four [8]. In addition to the use of social media, pervasive and ubiquitous computing and mobile networks have also dramatically increased the speed of communication. It also has revolutionised the access to healthcare information. In result, patients are sometimes not accepting doctors' or dentists' recommendations without doing their own research online [2], to find a trustworthy source. Indeed, if patients fail to have their needs (questions, diagnosis, and understanding) fulfilled through direct communication with doctors or dentists, they would search in Internet $[9,10]$. It has become a better source of information than the traditional healthcare providers. The attitude and behaviour of patients has changed due to ubiquitously available online information [11,12]. A survey indicated $81 \%$ of adult users have used Internet for health information and acknowledged that the Internet is the most widely used source for health information ahead of doctors, friends and families [13].

However, is the online data accurate? Search engines cannot provide answers to this question [13]. Not only the content but the source of the information is also critical while measuring trust. Classifying the information based on trust is one of the 
methods to overcome the colossal problem of information overloading in the Internet. Recommendation systems have been gaining popularity to do so. Traditionally two popular methods, content-based filtering (CB) and collaborative filtering (CF) are used. CB analyses description of items or individuals that have been rated by the user. A good example of this method is matchmaking sites, through which list of potential dates will be suggested based on the preferences chosen in the profile. However, the recommendation system like Amazon is not only using CB method but they analyse the users' similarity based on ratings and their profile (demographic information, history of buying etc.) and recommends products based on other users' behaviour. This method is known as collaborative filtering (CF). Both methods have limitations, $\mathrm{CB}$ does not consider the target users' similarity information whereas $\mathrm{CF}$ does not explicitly consider the content [14]. CF is one of the most popular methods used in recommendation systems, but has many challenges [14-16]. Cold start problem emanates when a new item or individual is added to the system and no user ratings exist in the initial phase. Scalability problem arises when the number of users and items rises exponentially. Shilling attacks are caused by biased behaviours such as making positive comments on own items and negative remarks to others. Data Sparsity is caused due to high volume of items/goods to recommend. Gray sheep symptom is when a user's preference is isolated and not similar to any other users in the system.

Main goal of recommendation systems is to suggest a list, based on knowledge extracted from consumers' previous behaviours. Recommendation systems have been using both CF and CB methods, referred as hybrid method. In this research, we will use hybrid method by analysing subjective characteristics of both patients and dentists. Trustworthiness is a critical factor while choosing a dentist due to the nature of invasive treatment.

\section{$3 \quad$ Trust and Personalisation from User Profiling}

Trust is an important sociological concept which depends on many factors such as past experiences, beliefs, values, tastes, personalities, opinions, rumours, influences and so on. It is defined and researched in various areas such as psychology, sociology, business, science, philosophy [17]; with many different meanings (contexts). In almost all contexts, trust is implied as a judgment in precarious situation that trustee will act in the best interest of truster [18]. Information which is useful and trustworthy in one context may not be useful in another context [5].

In dental treatment, trust emerges from an experience of interaction between patients and dentists; therefore the source of trust is an actual experience, referred as direct trust [17]. When the experience is transcribed in the web, it takes another form. It becomes a part of social trust, presented as a collection of experiences of many patients together. Trust usually can be looked at from two different aspects: functional (trust in performance) and referral (trust in recommendation) [19, 20]. Two patients may have different opinion about the trustworthiness of a dentist, despite the same behaviour and treatment by the same dentist. Therefore, personalisation of trust from subjective characteristics is important in social network environment, and it has been 
overlooked [5, 21]. This research focuses on subjective characteristics from user profiles to personalise the level of trust and match patients with suitable dentists, which will eventually increase trustworthiness in the recommendation system.

Traditional cues such as body language, tone of voice, facial expression and postures are present in face to face communications. But they are missing in the online communication, therefore, pertinent user profiling with subjective characteristics is critical to measure a level of trust precisely. In this research, we postulate that more accurate the profiles of both patients and dentists are, the greater chance to match a suitable dentist within a given time and place.

\section{$4 \quad$ Proposed Framework}

The proposed trust dependent framework for dynamic dental care recommendation system is shown in Figure 1.

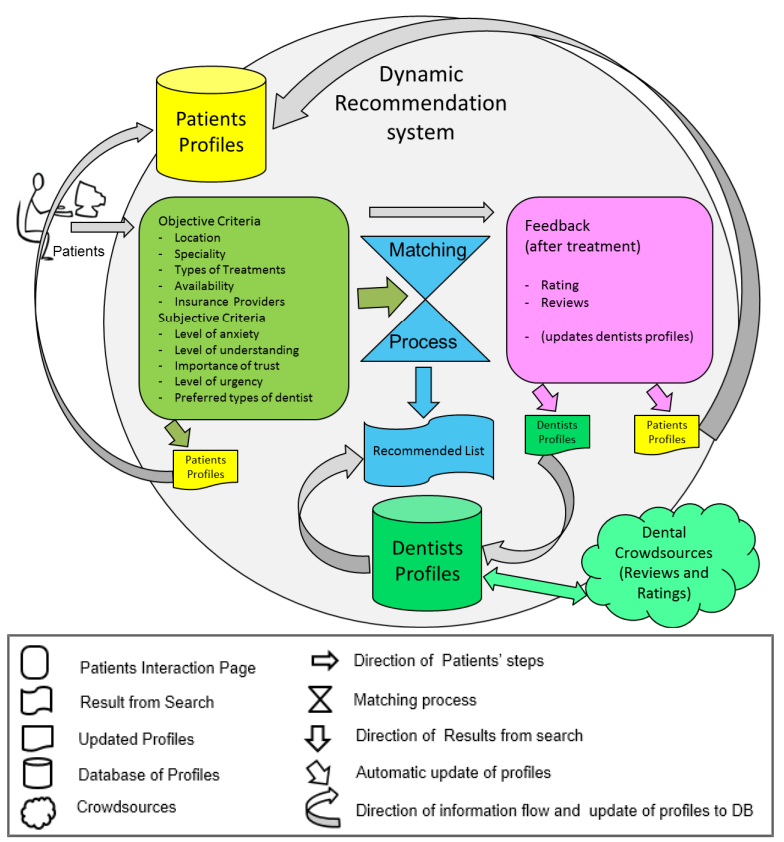

Fig. 1. Proposed profile-based trust framework for dentists recommendation system

Initially, patients will choose objective criteria such as location, specialist, treatment, availability and insurance providers. Subjective criteria such as attitudes, feelings, and perceptions would be determined by widely available standard personality tests. Based on the criteria, the system would provide a list of dentists (recommended list) available in the location with a number of reviews and aggregated ratings from the crowdsources. From the recommended list, the patient will choose a dentist for 
their treatment. After the treatment, patient will rate and write reviews about the interaction with the dentist. This information would update dentist's profile. Updating the information in real-time environment, for both patients and dentists, would benefit many other potential patients in future for the system.

For the purpose of this research, we have crawled dental reviews and ratings information from popular dental reviews sites: DrOogle ${ }^{1}$ and $\mathrm{Yelp}^{2}$ in the US. Separate reviews files are created for major cities in the US, for example, New York, Los Angeles, Chicago, and so on. The reviews for the dentists are analysed, by using text mining techniques to determine types of dentists.

\section{$5 \quad$ Trust Measurement from User Profiling and Crowdsources}

Trust plays a dynamic role in what people do in their daily activities. With an increased use of Internet, trust has been perceived in the form of security, privacy, credibility of source, quality of information, quality of systems and many more. Majority of trust related studies have been focusing on trends of behaviour and less on human factors such as personality, attitudes and perception. In this research, we focus on subjective characteristics of both patients and dentists to derive a level of trust. Majority of HSNs allow users to create, share and retrieve information and retain personal data online. Subjective characteristics such as attitude or perception of users are not available on the sites. Not only retrieving subjective characteristics of patient is challenging but privacy provision and anonymity adds even more complexity.

In this research, we source subjective characteristics of patients by asking directly to them. Based on ontology, natural way of being, people's attitudes, behaviours and perceptions have been studied from a very long time. In 1928, William Marston described that people show their emotions through attitudes and behaviours, using mainly four types of behaviour: Dominance (D), Inducement (I), Submission (S), and Compliance (C), referred as DISC personality test in modern days. Furthermore, extensive list of behaviours which fall into the categories of DISC are listed here [22].

- Dominance (D): direct, outspoken, decisive, assertive, competitive, ambitious and time-conscious.

- Inducement (I): influencer, friendly, talkative, expressive, attention-seeking, optimistic, outgoing and people-oriented.

- Submission (S): stable, consistent, good listener, patient, team player, need time to adjust, peacemaker and family-oriented.

- Compliance (C): competent, compliant, logical, analytical, perfectionist, organised, data driven, observant and detail-oriented.

DISC personality test is one of the methods to determine subjective criteria for patients and used in this study. There are many other methods available and the system will cater to use other methods as well. Analysing and finding out a type of patient and dentist is a critical process while matching for the recommendation systems.

\footnotetext{
${ }^{1}$ www.doctoroogle.com

${ }^{2}$ www.yelp.com
} 
For example, dental educator, Cathy Jameson [23] stated that qualification and expertise of dentist would be important for D type of patient and they would not like socialising as much, whereas, I type of patient would prefer discussing in a friendly manner.

The online survey we conducted in early stage of this research showed quality, reliability and even personality of dentist is important while choosing a dentist as shown in the figure 2 below. In this research, we have now taken the terms patients use to describe their dentists, as trust elements for dentists.

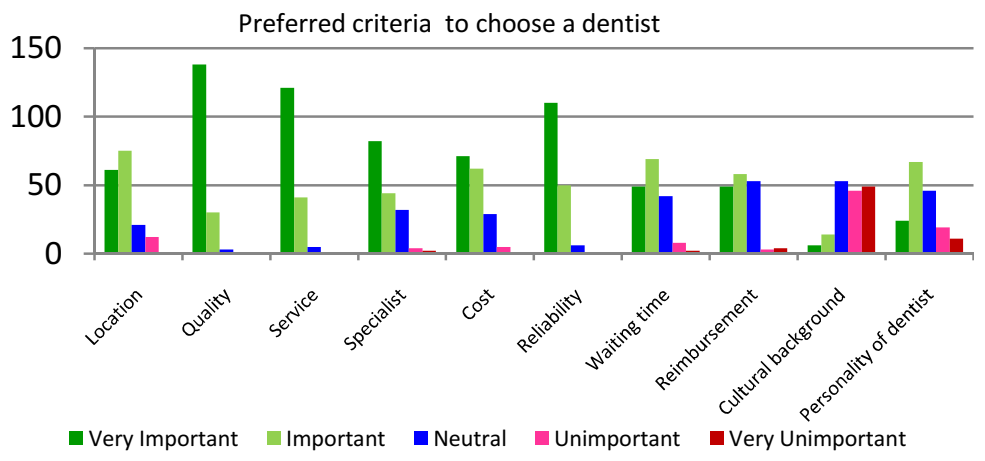

Fig. 2. Preferred criteria to choose a dentist

Question is "What combination of trust elements does the most trusted dentist constitute for a particular 'type of patient'?" Although different measuring criteria for ratings are used amongst different dental review sites [24], most of the sites allow patients to provide reviews about their experience after the dental visits. From two top US dental reviews sites: DrOogle and Yelp, we have analysed terms used to describe their dentists when the patients are satisfied or not satisfied. The terms are listed in the table 1 below. Based on the frequencies of terms used by patients for a typical dentist, type of dentist is determined. For example, if a term 'friendly' is used by many patients to describe a specific dentist, the dentist would be classified as a 'friendly' dentist.

Table 1. Trust elements affecting positively and negatively to dentists

\begin{tabular}{|l|l|l|}
\hline \multicolumn{2}{|c|}{ Trust elements affecting positively to Dentists } & Affecting negatively \\
\hline Friendly & Explains well & Rude \\
\hline Caring & Recommended by many & Rushed \\
\hline Professional & Quality of service & Poor manner \\
\hline Experienced & Reliable & Aggressive \\
\hline Knowledgeable & Good personality & \\
\hline
\end{tabular}

To determine the best combination of trust elements for a type of patient, an online survey and focus group will be conducted. Many researchers in dental area have identified and mentioned that caring, compassion, thoughtful and supportive dentists are preferred by patients $[25,26]$. Some others $[27,28]$ pointed out importance of 
expertise and knowledge sharing from dentists to the patients. These terms are also used to describe the patients in the reviews.

\section{Conclusion and Future Work}

This paper highlights that trust elements derived from subjective characteristics such as personality, attitudes, and perception of both patients and dentists, can enhance the suitability of matching while recommending a dentist to a patient. It proposes profilebased trust dependent framework for dynamic dental care recommendation systems. This framework could also be used in other domains as trust derived from different personality, attitude and perception, plays a vital role in dynamic recommendations systems. In the dental care, recommendation systems are still in their infancy and social elements of trust are not incorporated in the existing dental review sites. By integrating subjective characteristics, the effectiveness of recommendation systems would be increased and would provide benefits to the dental society. Collective intelligence from dental crowdsources is a major source for recommendations, but adding subjective criteria for patients and dentists, will enhance the matching process. In the near future, matching rules would be drawn through online questionnaires to general public (as a patient to a dentist) and dentists. The methods of online survey and focus group as well as analysis of dental reviews data will be explored further in the future. A recommendation tool has been developed to test the matching rule with a real reviews data available from the popular dental reviews sites. Eventually, the recommendation system would be a trusted source to find a suitable dentist.

\section{References}

1. Bhuiyan, T., Xu, Y., Josang, A.: State-of-the-Art Review on Opinion Mining from Online Customer's Feedback. In: The 9th Asia-Pacific Complex Systems Conference, Tokyo, Japan, November 4 -7 (2009)

2. Ratzan, S.C.: Our New "Social" Communication Age in Health. Journal of Health Communication 16(8), 803-804 (2011)

3. Swan, M.: Emerging patient-driven health care models: an examination of health social networks, consumer personalized medicine and quantified self-tracking. International Journal of Environmental Research and Public Health 6(2), 492-525 (2009)

4. Lilien, L., Bhargava, B.: Trading privacy for trust in online interactions. Idea Group (2008)

5. Jøsang, A., Quattrociocchi, W., Karabeg, D.: Taste and trust. In: Wakeman, I., Gudes, E., Jensen, C.D., Crampton, J. (eds.) Trust Management V. IFIP AICT, vol. 358, pp. 312-322. Springer, Heidelberg (2011)

6. Gray, E., Seigneur, J.-M., Chen, Y., Jensen, C.: Trust propagation in small worlds. In: Nixon, P., Terzis, S. (eds.) iTrust 2003. LNCS, vol. 2692, pp. 239-254. Springer, Heidelberg (2003)

7. Shu, W., Chuang, Y.H.: The perceived benefits of six-degree-separation social networks. Internet Research: Electronic Networking Applications and Policy 21(1), 26-45 (2011)

8. Barnett, E.: Facebook cuts six degrees separation to four. In: The Telegraph (November 22, 2011),

http://www.telegraph.co.uk/technology/facebook/8906693/

Facebook-cuts-six-degrees-of-separation-to-four.html (2012) 
9. Hou, J., Shim, M.: The Role of Provider-Patient Communication and Trust in Online Sources in Internet Use for Health-Related Activities. Journal of Health Communication 15(S3), 186-199 (2010)

10. Tustin, N.: The role of patient satisfaction in online health information seeking. Journal of Health Communication 15(1), 3-17 (2010)

11. Pickens, J.: Attitudes and perceptions. In: Borkowski, N. (ed.). Organizational Behavior in Health Care, ch. 3, Michael Brown, USA, pp. 43-76 (2005)

12. Ferguson, M.J., Bargh, J.A.: Beyond the attitude object: Automatic attitudes spring from object-centered-objects. In: Wittenbrink, B., Schwarz, N. (eds.) Implicit Measures of Attitudes, pp. 216-246 (2007)

13. Moturu, S., Liu, H.: Quantifying the Trustworthiness of Social Media Content: Content Analysis for the Social Web. LAP Lambert Academic Publishing (2010)

14. Su, X., Khoshgoftaar, T.M.: A Survey of Collaborative Filtering Techniques. Hindawi Publishing, Advances in Artificial Intelligence (2009)

15. Walter, F.E., Battiston, S., Schweltzer, F.: A model of trust-based recommendation system on a social network. Auton Agent Multi-Agent System 16, 57-74 (2008)

16. Lopez-Nores, M., Blanco-Fernandez, Y., Pazos-Arias, J.J., Diaz-Redondo, R.P.: PropertyBased Collaborative Filtering: A New Paradigm for Semantics-Based, Health-Aware Recommender Systems. In: Fifth International Workshops on Semantics Media Adaptation and Personalization (2010)

17. Dokoohadi, N., Matskin, M.: Personalizing Human Interaction through Hybrid Ontological Profiling Cultural Heritage Case Study. In: International Workshop on Semantic Web Applications and Human Aspects (SWAHA), Collocated with 3rd Asian Semantic Web Conference (ASWC), pp. 133-140 (2008)

18. Goudge, J., Gilson, L.: How can trust be investigated? Drawing lessons from past experience. Social Science \& Medicine 61(7), 1439-1451 (2005)

19. Victor, P., De Cock, M., Cornelis, C.: Trust and Recommendations. In: Ricci, F., Rokach, L., Shapira, B., Kantor, P.B. (eds.) Recommender Systems Handbook, 1st edn. (2011)

20. Josang, A., Pope, S.: Semantic constraints for trust transitivity, pp. 59-68. Australian Computer Society, Inc. (2005)

21. Golbeck, J.: Computing and applying Trust in web-based Social Networks, University of Maryland, Maryland, USA (2005)

22. DISC Insights, http : / /www. discinsights . com

23. Meyer, E.: Meeting the needs of difficult dental patients, clear communication and careful preparation can help moderate stressful situations. Inside Dental Assisting 10(5) (September/October 2012)

24. Pradhan, S., Gay, V., Nepal, S.: Social Networking and Dental Care: State of the Art and Analysis of the Impact on Dentists, Dental Practices and their Patients. In: BLED 2013 Proceedings, Paper 22 (2013)

25. Sbaraini, A., Carter, S.M., Evans, W., Blinkhorn, A.: Experiences of dental care: What do patients value? BMC Health Services Research 12, 177 (2012)

26. Yarascavitch, C., Regehr, G., Hodges, B., Haas, D.A.: Changes in Dental Student Empathy During Training. Journal of Dental Education 73(4), 509-517 (2009)

27. Merijohn, G.K., Bader, J.D., Frantsve-Hawley, J., Aravamudhan, K.: Clinical decision support chairside tools for evidence-based dental practice. Journal Evid. Base Dent. Pract. 8, 119-132 (2008)

28. Mettes, T.G., van der Sanden, W.J.M., Mokkink, H.G., Wensing, M., Grol, R.P.T.M., Plasschaert, A.J.M.: Routine oral examinations in primary care: Which predictors determine what is done?, A prospective clinical case recording study. Journal of Dentistry 36, 435-443 (2008) 